\title{
Medindo Associação Implícita com o FreeIAT em Português: Um Exemplo com Atitudes Implícitas frente ao Poliamor
}

\author{
Measuring Implicit Association with the FreeIAT in Portuguese: \\ An Example with Implicit Attitudes toward Polyamory
}

\author{
Valdiney V. Gouveia*, ${ }^{*}$, Luis Augusto de Carvalho Mendes ${ }^{b}$, Sandra Elisa de Assis Freire ${ }^{c}$, \\ Leogildo Alves Freires ${ }^{d} \&$ Larisse Helena Gomes Macêdo Barbosa ${ }^{a}$ \\ ${ }^{a}$ Universidade Federal da Paraíba, João Pessoa, PB, Brasil, \\ ${ }^{b}$ Faculdade Maurício de Nassau, João Pessoa, PB, Brasil, ${ }^{~}$ Universidade Federal do Piaui, Teresina, PI, Brasil \\ \& ${ }^{d}$ Universidade Federal de Roraima, Boa Vista, RR, Brasil
}

\begin{abstract}
Resumo
O Teste de Associação Implícita (TAI) tem se mostrado relevante na medição de diversos construtos psicológicos. Entretanto, são escassos os estudos que o consideram no Brasil. Este artigo objetivou introduzir um programa gratuito (FreeIAT) para sua realização, exemplificando com uma medida de atitudes frente ao poliamor. Participaram da pesquisa 50 estudantes universitários com idade média de 22 anos (62\% mulheres), que responderam a uma escala de atitudes explícitas frente ao poliamor e ao TAI Monogamia-Poliamor. Os resultados mostraram correlação negativa entre a medida implícita (bloco de latência incongruente) e a explícita de atitudes frente ao poliamor, indicando que quanto maior a aceitação do poliamor, menor o tempo de associação entre os estímulos (palavras positivas e poliamor). Não se observou correlação entre o Escore D e as atitudes explícitas frente ao poliamor, sugerindo que pessoas favoráveis ao poliamor não necessariamente são contrárias à monogamia. Concluiu-se que o FreeIAT possui uma interface simplificada, atendendo às variações do TAI clássico e permitindo aplicação coletiva. Além disso, o fato de ser um programa com código aberto e gratuito tem o potencial de incentivar pesquisas com medidas implícitas em culturas lusófonas.

Palavras-chave: FreeIAT, TAI, medidas implícitas, atitudes, poliamor.
\end{abstract}

\begin{abstract}
Even the Implicit Association Test (IAT) has been shown to be relevant in measuring various psychological constructs there are few studies considering it in Brazil. This paper aims to introduce a free program (FreeIAT) to perform IAT, illustrating it with a measure of attitudes toward polyamory. Participants were 50 undergraduate students with mean age of 22 years ( $62 \%$ women) who answered an explicit scale attitude toward polyamory and the Monogamy-Polyamory IAT. Results showed a negative correlation between implicit (latency of incongruent block) and explicit attitudes measures toward polyamory, indicating that the greater the acceptance of polyamory, the lesser time to association between the stimuli (positive words and polyamory). There was no correlation between the D Score and the explicit attitudes toward polyamory, suggesting that people in favor of polyamory are not necessarily opposed to monogamy. In conclusion, the FreeIAT shows a streamlined interface, following the variations of the classic IAT and allowing collective application. Moreover, the fact of being a free program with open source it has the potential to encourage research on implicit measures in Lusophone cultures.

Keywords: FreeIAT, IAT, implicit measures, attitudes, polyamory.
\end{abstract}

Manuais de psicologia social mostram que as medidas explícitas, que requerem atenção consciente do respondente, atualmente dominam a literatura da área (Gawronski \& Bodenhausen, 2006; Maio \& Haddock, 2010). Entretanto, observa-se uma demanda crescente por medidas implícitas,

* Endereço para correspondência: Centro de Ciências Humanas Letras e Artes, Departamento de Psicologia, Universidade Federal da Paraíba, Campus I, Cidade Universitária, s/n, João Pessoa, PB, Brasil 58051-900. E-mail:vvgouveia@gmail.com que não dependem da atenção consciente do respondente, sendo suas respostas automáticas e espontâneas (Gouveia, Athayde, Mendes, \& Freire, 2012; Nosek, Hawkins, \& Frazier, 2011; Pimentel, Torres, \& Günther, 2011). De fato, uma das vantagens das medidas implícitas é diminuir ou anular o efeito da desejabilidade social, comum às pesquisas de autorrelato (Gouveia, Guerra, Souza, Santos, \& Costa, 2009). Seisdedos (1996), por exemplo, afirma que as pessoas são sujeitas às normas sociais e tendem a dissimular suas respostas, apresentando-se de forma socialmente aceitável, assegurando a autoafirmação e a 
aceitação grupal. Este viés tende a distorcer as respostas em uma direção favorável, necessitando de um controle (Furnham, 1986). Uma forma de controlar esta tendência é por meio de mensurações implícitas ou automáticas, que pressupõem a diminuição da reatividade do respondente (Lane, Banaji, Nosek, \& Greenwald, 2007; Pruett \& Chan, 2006).

Processos automáticos ou implícitos são aqueles que acontecem de forma espontânea, sem controle, intenção ou interferência de qualquer atividade mental consciente (Fazio \& Olson, 2003). Bargh (1994) define o automatismo com base em quatro características: consciência, intenção, eficiência e controle cognitivo, sendo automático o processo que tenha ausência de qualquer uma destas características. Com o fim de operacionalizar os processos automáticos em variáveis de pesquisa, destacam-se dois tipos de recursos: o priming (Fazio, 1995) e a latência de tempo de resposta, destacando-se o Teste de Associação Implícita (TAI; Greenwald, Mcghee, \& Schwartz, 1998). Gouveia et al. (2012) acrescentam que medidas de respostas neurofisiológicas têm dado igualmente uma contribuição para os processos automáticos ou implícitos, como as obtidas com técnicas de neuroimagem, testes de resposta galvânica da pele, verificação de atividade eletromiográfica e movimentação dos olhos ou resposta pupilar. Estas têm sido alternativas de medidas implícitas por serem respostas neurais ou metabólicas, dependentes de órgãos corporais, não estando comumente sob o controle dos respondentes (Wittenbrink \& Schwarz, 2007).

Em razão de suas potencialidades, os testes de associação implícita vêm sendo amplamente usados em Psicologia, sendo considerados úteis e adequados para medir diversos construtos (Bassili \& Brown, 2005; Maio \& Haddock, 2010; Wittenbrink \& Schwarz, 2007). A propósito, em busca realizada no PsycNET (2012), inserindo como expressão-chave "implicit association test", foi possível encontrar 1.868 publicações, destacando-se aquelas sobre processos de associação, memória, autoestima, atitudes estereotipadas e validação de testes. Apesar deste número de publicações no cenário internacional, em buscas realizadas em sites acadêmicos nacionais (Google Acadêmico, 2012; Periódicos Capes, 2012), utilizando a mesma expressão-chave em português, foram listados 38 documentos, entre trabalhos de conclusão de curso (TCC), dissertações, teses e artigos. Isso revela que ainda é pequena a produção na área em língua portuguesa tratando com medidas implícitas.

Com o propósito de incentivar o uso do Teste de Associação Implícita (TAI) em países lusófonos, planejou-se o presente artigo com o objetivo de adaptar para o português o programa FreeIAT (Meade, 2009). No caso, mostrando como funciona, oferecendo um exemplo específico de pesquisa para avaliação de atitudes implícitas frente ao poliamor. Antes, entretanto, demanda-se descrever sumariamente o TAI.

\section{Teste de Associação Implícita}

Com base na análise da latência de resposta, Greenwald et al. (1998) propuseram o Teste de Associação Implícita (TAI), em que a exposição a um objeto de avaliação atitudinal pode ativar reações mentais automáticas que afetam a velocidade das respostas subsequentes. Assim, esta técnica tenta obter resultados que estejam fora da intencionalidade do respondente, focando no acesso de atitudes que ele pode ser relutante ou incapaz de expressar. Greenwald e Banaji (1995) defendem que tais atitudes resultam de experiências passadas das quais a pessoa tem pouca ou nenhuma consciência, mas que influenciam processos cognitivos, afetivos e comportamentais, com carga positiva ou negativa, frente a um objeto atitudinal. Portanto, o TAI mede atitudes implícitas avaliando a latência de resposta da associação entre uma categoria-alvo e uma categoria de atributos.

Pensado para ser executado em computador, o TAI clássico mede a diferença entre as associações das categorias-alvo e dos atributos em frações de milissegundos (ms; Greenwald et al., 1998). A ideia básica é que atitudes que estejam mais latentes são acionadas de forma mais rápida e com menor quantidade de erros (Lane et al., 2007). Neste sentido, os participantes são expostos a uma série de estímulos (palavras, números ou imagens) dispostos no centro da tela do computador, devendo associá-los às categorias que aparecem nos lados esquerdo e direito da tela. Para fazer a associação, eles têm que pressionar a chave da esquerda (geralmente a tecla "E"), quando o estímulo pertencer à categoria da esquerda, ou a da direita (geralmente tecla "I"), quando pertencer à categoria da direita. $\mathrm{Na}$ instrução, cada respondente é orientado a executar o procedimento o mais rápido possível, cometendo a menor quantidade de erros (Stüttgen, Vosgerau, Messner, \& Boatwright, 2011).

O procedimento é frequentemente composto por cinco blocos, sendo três de ensaio e dois de teste. No Bloco 1 de ensaio, os participantes são expostos a estímulos para classificá-los nas categorias-alvo correspondentes (e.g., rosas e tulipas na categoria flores, abelhas e vespas na categoria insetos). No segundo bloco de ensaio, é solicitada a associação de estímulos com as categorias de atributos (e.g., alegria e paz na categoria palavras positivas, ódio e triste na categoria palavras negativas). O Bloco 3 é a primeira fase do teste de associação, em que aparecem os emparelhamentos de categorias (e.g., lado esquerdo $=$ flores + palavras positivas; lado direito $=$ insetos + palavras negativas) e os participantes têm que associar os estímulos de acordo com os emparelhamentos das categorias. O Bloco 4 é um novo ensaio em que os estímulos das categorias-alvo são novamente apresentados aos participantes, porém havendo inversão com respeito ao Bloco 1. Assim, a categoria-alvo que inicialmente foi apresentada do lado direito, passa para o lado esquerdo e vice-versa. Por fim, no Bloco 5 é a segunda fase do teste, 
Gouveia, V. V., Mendes, L. A. C., Freire, S. E. A., Freires, L. A. \& Barbosa, L. H. G. M. (2014). Medindo Associação Implícita com o FreeIAT em Português: Um Exemplo com Atitudes Implícitas frente ao Poliamor.

onde os emparelhamentos do Bloco 3 são invertidos (e.g., lado esquerdo $=$ insetos + palavras positivas; lado direito $=$ flores + palavras negativas).

Os Blocos 3 e 5 medem a velocidade de associação entre um objeto atitudinal e os atributos. Portanto, indivíduos que têm uma avaliação positiva frente às flores associam mais rapidamente as palavras estímulos desta categoria com as palavras positivas, fazendo-o de forma mais lenta em relação aos insetos. O bloco com menor média de tempo de associação (menor latência de resposta) é denominado bloco congruente, enquanto que aquele com maior média de tempo de associação (maior latência de resposta) é definido como bloco incongruente. Na literatura, estes termos podem ser definidos também como associações compatíveis e incompatíveis, respectivamente (Lima, Machado, Ávila, Lima, \& Vala, 2006).

O modelo convencional de medir a associação implícita é pela diferença entre o valor do bloco incongruente (A) e congruente (B) $[\mathrm{TAI}=\mathrm{A}-\mathrm{B}]$ (Greenwald et al., 1998). Esta diferença entre a média de latência de resposta dos dois blocos é considerada como a força das associações subjacentes (Lemm, Lane, Sattler, Khan, \& Nosek, 2008). Devido à variabilidade nos escores provenientes da diferença habilidade de execução do teste, Greenwald, Nosek e Banaji (2003) desenvolveram o Escore D (GNB Score), que é o resultado da diferença entre os blocos incongruentes e congruentes (escore convencional) dividido pelo desvio-padrão do indivíduo de todas as latências de respostas, nos dois blocos.

O Escore $D$ varia de -2 a +2 , onde valores negativos indicam uma atitude contrária ao objeto de pesquisa e os positivos expressam uma atitude favorável ao objeto de estudo (Cai, Sriram, Greenwald, \& McFarland, 2004; Greenwald et al., 2003; Stüttgen et al., 2011). Quanto à força da associação, valores de Escore $D$ até 0,15 indicam não haver uma preferência nítida, entre 0,16 e 0,35 evidenciam uma associação fraca, de 0,36 a 0,65 uma preferência moderada e acima de 0,65 é considerada uma preferência forte. Estes índices servem tanto para valores positivos quanto negativos (Nosek, Greenwald, \& Banaji 2007; Sriram \& Greenwald, 2009).

É importante ressaltar que o TAI tem sido ampliado para diferentes públicos, como crianças com 4 anos de idade (Preschool Implicit Association Test [PSIAT]; Cvencek, Greenwald, \& Meltzoff, 2011), de 6 a 10 anos (Cvencek, Meltzoff, \& Greenwald, 2011) e mesmo adolescentes (Baron \& Banaji, 2006). Também vem sendo empregado para estudos com comedores compulsivos (Ayres, Prestwich, Conner, \& Smith, 2011) e trabalhadores (Leavitt, Fong, \& Greenwald, 2011). Com relação aos construtos pesquisados, têm sido avaliados o bem-estar (Walker \& Schimmack, 2008), o preconceito frente ao uso de drogas (Hippel, Brener, \& Hippel, 2008) e a personalidade implícita (Schnabel, Asendorpf, \& Greenwald, 2008), por exemplo. A propósito da gama de possibilidades de aplicação do TAI, Nosek et al. (2011) listaram 20 procedimentos de mensuração implícita, cabendo destaque para algumas de suas variações: Single Categorie IAT (SC-IAT; Karpinski \& Steinman, 2006), Go/No-Go (Nosek \& Banaji, 2001) e Filtering Unconscious Matching of Implicit Emotions (FUMIE; Mori, Uchida, \& Imada, 2008).

Apesar de evidente ascensão, as medidas implícitas sofrem críticas quanto à sua aplicabilidade, mensurabilidade, confiabilidade, poder preditivo e relação com outras medidas (Arkes \& Tetlock, 2004; Blanton \& Jaccard 2006; Fiedler, Messner, \& Bluemke, 2006; Steffens, 2004). Porém, Jost et al. (2009) analisaram pesquisas publicadas entre os anos 2001 e 2008 e verificaram que participantes como estudantes, enfermeiros, médicos, policiais e recrutadores de emprego apresentaram preconceitos implícitos em relação à raça, etnia, nacionalidade, sexo e status social que foram condizentes com suas atividades, o que reforça a adequação desta técnica de mensuração. A respeito do impacto do TAI, em levantamento dos 15 anos desde a publicação do primeiro artigo sobre este tema, Nosek et al. (2011) definiram o período como a "era das medidas implícitas", uma vez que foram desenvolvidos mais de 20 procedimentos de mensuração, destacando pesquisas com atitudes, estereótipos, autoestima e autoconceito, envolvendo áreas diversas, como consumo, saúde mental e comportamento político. Pimentel et al. (2011) apontam que as críticas e os novos procedimentos têm possibilitado o aprimoramento e a divulgação deste tipo de medida. Neste âmbito, a proposta do FreeIAT oferece uma contribuição importante a respeito (Meade, 2009).

\section{Adaptação do FreeIAT para o Português}

Este é um programa grátis e de código aberto (open source), que se apresenta como uma alternativa aos programas comerciais para execução do TAI (e.g., DirectRT, Inquisit, Superlab). Portanto, seu objetivo é promover a utilização desta técnica, tornando-a acessível para os pesquisadores lusófonos, evitando gastos adicionais. Ele foi construído no Visual Basic Express 2008, que é disponibilizado gratuitamente pela Microsoft e roda em todas as plataformas Windows com o Microsoft.NET v.2.0 ou mais atual (Meade, 2009). Originalmente escrito em inglês, é um programa distribuído sob Licença Pública Geral (versão 3), determinando que todos os recursos derivados dele sejam disponibilizados gratuitamente. O FreeIAT é de fácil utilização e possui uma tela única de menus, com opções para a execução direta do TAI ou para configurar novos procedimentos. O programa fornece um arquivo de resultados completo, que inclui um sumário dos estímulos administrados, as respostas dos participantes e o tempo demandado em responder cada etapa do teste. Além disso, é fornecido o Escore D (GNB Score), na linha dos procedimentos recomendados por Greenwald et al. (2003).

Pesquisas em sites de busca de produções acadêmicas (Google Acadêmico, 2012; Periódicos Capes, 2012) mostraram que estudos recentes com medidas implícitas têm utilizado o FreeIAT. Por exemplo, a pesquisa de Hartmana e Newmarka (2012) que analisou a relação de medidas 
explícitas sobre religião e política com medidas implícitas (TAI) que relacionaram o presidente estadunidense Barack Obama com o Islamismo; Clark e Meade (2012) estudaram a validade e falseabilidade do Teste de Associação Implícita de Integridade Autopercebida; e Wright e Meade (2011) o utilizaram para conhecer a validade preditiva de um TAI medindo a capacidade de realização de tarefas alternadas. Por fim, mais recentemente Wright e Meade (2012) verificaram a relação entre os escores do TAI e aqueles de testes de habilidades cognitivas. Nesta pesquisa foram testadas duas metodologias alternativas de pontuação: o Escore D e o escore convencional.

Diante das vantagens do TAI e da disponibilidade de um programa gratuito (FreeIAT), com potencial para estimular os estudos na área de medidas implícitas, decidiu-se adaptá-lo, produzindo uma versão em português. Descreve-se a seguir o processo de sua tradução, mostrando, posteriormente, sua configuração, seu modo de execução e os resultados proporcionados. Finalmente, apresenta-se uma pesquisa empírica que detalha seu uso.

\section{Tradução}

Na tradução para o português foi utilizado o procedimento de retrotradução (back translation), em que os textos originais do programa, em inglês, foram traduzidos para o português por dois profissionais de psicologia bilíngues e, posteriormente, retraduzidos para o inglês por outros dois psicólogos bilíngues; todos tinham conhecimento de utilização do TAI. Confrontadas as duas versões em inglês e comparadas com as traduções em português, chegou-se à versão final, que foi implementada a partir do Visual Basic Express 2008. No caso, alteraram-se apenas os textos do programa original sem que os procedimentos de análise e aferição do TAI fossem modificados. O processo de tradução foi autorizado pelo autor do FreeIAT (A. W. Meade, comunicação pessoal, 20 de dezembro, 2011). O leitor interessado poderá obter uma cópia desta nova versão escrevendo para um dos autores deste artigo, conforme endereços disponíveis em sua página web (http:// vvgouveia.net).

\section{Instruções de Instalação e Uso}

Uma vez obtido o diretório compactado com os arquivos do FreeIAT, sua instalação será praticamente automática. Concretamente, bastará descompactá-lo em uma pasta e clicar no arquivo executável (IATapp1.exe). Quando o programa for iniciado, aparecem as opções de iniciar, ajuda, configuração, atualização da versão e a tecla sair. Neste instante, interessa a opção configuração, pois permitirá criar ou alterar a nova medida do TAI.

\section{Configuração da Medida}

Clicando no botão configuração é solicitado o título do teste e perguntado se são utilizadas imagens no procedimento. O título cadastrado aparecerá na tela de instruções do TAI quando este for executado. Em seguida é apresentada a tela para o cadastramento das duas primeiras categorias de atributos. Na coluna da esquerda, cadastram-se o nome da primeira sequência e, no quadro abaixo, os estímulos, separando-os com enter. Da mesma forma ocorre o cadastramento da coluna da direita. A literatura indica quatro ou mais estímulos como número ideal para cada categoria, sendo aceitável um mínimo de dois (Nosek, Greenwald, \& Banaji, 2005).

$\mathrm{Na}$ tela seguinte são cadastradas as categorias-alvo ou condições; o cadastramento segue o mesmo procedimento da tela anterior. Ressalta-se a importância da ordem em que as categorias são cadastradas, pois os Escores D positivos indicam a associação entre a primeira sequência de palavras com a primeira condição ou categoria, enquanto que os Escores $D$ negativos expressam a associação entre a primeira sequência de palavras com a segunda condição ou categoria. Dito de outro modo, no caso dos Escores $D$ positivos, estes indicam a congruência esperada entre a categoria e os estímulos ou palavras respectivas (por exemplo, a categoria amor com as palavras bom, alegre e maravilhoso); por outro lado, os Escores $D$ negativos sugerem a incongruência destas associações (por exemplo, a categoria amor combinada com palavras negativas, como ruim, triste e horrível).

Também é possível utilizar imagens como categorias-alvo, bastando informar o nome da figura com a extensão correspondente (e.g., “imagem1.jpg”). Neste caso, as imagens devem ser colocadas diretamente na pasta onde está o arquivo executável (IATapp1.exe). Caso deseje guardar as imagens em outra pasta, esta deve ser criada no diretório onde o arquivo executável está instalado. Após criar a pasta, basta informar, no quadro reversado para os estímulos, o nome da pasta seguido do nome da imagem, com a extensão respectiva (e.g., "pasta/imagem1. jpg"). Não existe um tamanho máximo para as imagens, porém a visualização dependerá da resolução do monitor. É aconselhável analisar o tamanho da imagem antes da aplicação dos testes, evitando que fique muito grande ou pequena; o ideal é que ela permaneça centralizada no monitor do computador. Arquivos com extensão jpg e gif já foram testados na versão em português, funcionando adequadamente.

No FreeIAT é possível configurar a quantidade de tentativas para cada um dos blocos de ensaios (1, 2 e 4) e testes de associação (3 e 5), requerendo apenas informá-las. Nosek et al. (2005) sugerem que nos blocos de treino (1, 2 e 4) um número entre 20 e 40 tentativas é o ideal, principalmente no Bloco 4, reduzindo o impacto da ordem do teste. Para os Blocos 3 e 5, 60 tentativas têm sido a opção mais usual. Greenwald et al. (2003) e Nosek et al. (2005) descrevem os Blocos 3 e 5 separados em duas etapas, sendo uma de ensaio com 20 tentativas e outra de teste com 40 tentativas, porém ambas etapas são computadas no escore final. Deste modo, tais etapas são combinadas sem diferenciação no cálculo do Escore D (Clark \& Meade, 2012; Wright \& Meade, 2011). Por fim, é solicitado o separador 
Gouveia, V. V., Mendes, L. A. C., Freire, S. E. A., Freires, L. A. \& Barbosa, L. H. G. M. (2014). Medindo Associação Implícita com o FreeIAT em Português: Um Exemplo com Atitudes Implícitas frente ao Poliamor.

das variáveis nos arquivos de resultado; orienta-se que na versão em português se utilize o espaço, pois a vírgula já é utilizada pelo Windows em português para separar os valores decimais.

Uma das facilidades oferecidas por este programa é a possibilidade de executar vários testes em um mesmo computador ou instalá-lo em várias máquinas, bastando para isso copiar a pasta onde ele se encontra e colocá-la em um novo local. Também é possível utilizá-lo a partir de um dispositivo móvel, como um pendrive, executando-o em qualquer computador com o Windows. Isso, certamente, facilita a aplicação de forma coletiva.

\section{Execução da Tarefa}

Para executar o teste é obrigatório entrar com uma identificação, que pode compreender qualquer combinação de números e letras, e clicar em iniciar. Na tela seguinte são apresentadas as instruções para a realização do teste e as categorias do TAI com os respectivos itens (estímulos). Após a leitura das instruções e identificação dos estímulos pelo participante, basta clicar na opção estou pronto para iniciar, que permitirá realizar o teste, ou em sair, que fará com que o respondente encerre a atividade, abandonando o programa.

Após a iniciação do teste, a primeira tela a ser apresentada é o treinamento com os estímulos das categorias-alvo; para iniciá-lo é necessário clicar na tecla barra de espaço. Finalizado o primeiro treino, é iniciado o treinamento com as categorias de atributos. Novamente é solicitado acionar a barra de espaço para iniciar o teste com o primeiro emparelhamento de categorias, constituindo-se no primeiro teste; na sequência, inicia-se o treinamento com as categorias-alvo de forma invertida. Posteriormente, é realizado o segundo e último teste, na ocasião com o emparelhamento invertido. Todos os comandos necessários para a sequência do TAI são exibidos pelo próprio programa.

\section{Arquivo de Dados e Resultados}

Para verificar os resultados, basta abrir o arquivo ScoresOnly.txt; uma opção é abrir o AllData.txt em que todos os dados do teste são armazenados. Contudo, o ScoresOnly.txt é mais utilizado por apresentar as informações principais do TAI, mostradas na seguinte sequência:

1. Identificação do participante;

2. Escore D (GNB Score; Greenwald et al., 2003);

3. Média corrigida do tempo de resposta (em ms) do Bloco 3 utilizando a substituição de item perdido;

4. Média corrigida do tempo de resposta (em ms) do Bloco 5 utilizando a substituição de item perdido;

5. Desvio padrão do tempo de resposta dos Blocos 3 e 5 juntos;

6. Média do tempo real de resposta (sem ajustes) do Bloco 3;

7. Média do tempo real de resposta do Bloco 5;

8. Escore $D$ computado utilizando apenas a primeira metade dos estímulos dos Blocos 3 e 5;
9. Escore $D$ computado utilizando apenas a segunda metade dos estímulos dos Blocos 3 e 5 (correlacionando os valores das colunas 8 e 9 consegue-se uma estimativa de consistência interna); e, finalmente,

10. Data e tempo de realização do TAI.

Para carregar os dados em programas estatísticos recorrentes [e.g., Excel, $P A S W$ e $R$ ], basta indicar a importação de um arquivo de texto com as variáveis delimitadas por espaço. Os valores decimais são separados por vírgula, logo esta opção deve ser descartada como delimitadora de dados; se o usuário desejar, poderá utilizar o ponto e vírgula (;).

Por fim, destaca-se a natureza intuitiva do programa, que permite ao usuário seguir facilmente os passos anteriormente indicados e implementar sua medida de associação implícita. Porém, como se comentou previamente, existe também uma opção de ajuda que poderá proporcionar-lhe auxílio; seus autores ainda disponibilizam um guia rápido de uso. Com o fim de mostrar a adequação deste programa, descreve-se a seguir um estudo sobre atitudes implícitas frente ao poliamor, procurando comparar os resultados desta medida implícita com aqueles de uma medida explícita sobre o mesmo construto.

\section{Atitudes frente ao Poliamor: Utilizando o FreeIAT}

O poliamor diz respeito a um tipo de relacionamento que recusa a monogamia como princípio ou necessidade, podendo ser definido como uma prática de amor de forma aberta, eticamente partilhada por várias pessoas; seus adeptos enfatizam mais o amor do que a sexualidade, preferindo, por exemplo, serem chamados de "poliamorosos" (Wolfe, 2005).

O termo poliamor é geralmente usado para se referir à prática de ter mais de um relacionamento íntimo, simultâneo, com o conhecimento e consentimento de todos os envolvidos (Anapol, 2010; Barker, 2005). Portanto, ele desafia os elementos do amor romântico que pauta a sociedade ocidental, cujo paradigma central das relações amorosas se pauta na ideia de considerar que o casal deve se relacionar com exclusividade entre si, primando pela intimidade e o compromisso (Gouveia, Carvalho, Santos, \& Almeida, 2013).

Um princípio destacado e cultivado no poliamor é o “compersion”, emoção que é oposta ao ciúme, uma vez que este é um dos maiores desafios emocionais para quem se propõe a viver este tipo de relacionamento. Compersion significa sentir prazer e alegria ao ver seu(sua) parceiro(a) sendo amado(a) por outra pessoa; ele é descrito como uma modalidade de sentimento de empatia (Anapol, 2010). O elemento central do poliamor não é a sexualidade, mas o afeto; ama-se a múltiplos parceiros, com consentimento dos envolvidos, o que exclui outras modalidades de relacionamento, a exemplo do swing (Barker, 2005). 


\section{Método}

\section{Delineamento e Hipóteses}

Tratou-se de estudo correlacional, de corte transversal, objetivando conhecer como e com que magnitude se correlacionam as medidas implícita e explícita de atitude frente ao poliamor. Estabeleceu-se a hipótese de que as pontuações destas medidas se correlacionariam entre si, indicando que quanto maior a pontuação na medida de atitude explícita favorável ao poliamor, menor o tempo para associar esta categoria com palavras positivas.

\section{Participantes}

Participaram 50 estudantes de uma universidade pública do Piauí com idade média de 22 anos $(D P=3,04)$, a maioria do sexo feminino (62\%), solteira (92\%), de classe média (64\%) e católica (84\%), com nível médio de religiosidade de 2,8 $[D P=1,26$; escala de resposta variando de 1 (Nada religioso) a 5 (Totalmente religioso)]. Tratou-se de uma amostra de conveniência, tendo participado as pessoas que, convidadas em sala de aula de disciplina de Psicologia, dispuseram-se a colaborar voluntariamente.

\section{Instrumentos}

Os participantes responderam um livreto com as seguintes partes:

Escala de Atitudes Frente ao Poliamor (EAFP). Esta medida explícita é formada por 12 itens presumivelmente distribuídos em dois fatores: relacionamento poliamorista, que indica atitudes favoráveis frente à possibilidade de uma relação afetiva poliamorista (e.g., Como ninguém manda no coração, é possível amar mais de uma pessoa ao mesmo tempo); e sentimento poliamorista, enfocando sentimentos favoráveis em relação à prática poliamorista [e.g., É prazeroso ver seu(sua) parceiro(a) envolvido(a) com outra pessoa quando essa relação envolve apenas o sexo]. Seus itens são respondidos em escala tipo Likert, de cinco pontos, variando de $\mathbf{1}$ (Discordo Totalmente) a 5 (Concordo Totalmente). Estes fatores apresentaram consistência interna (alfa de Cronbach) de 0,86 e 0,78, respectivamente.

TAI Monogania-Poliamor. É uma medida de atitudes implícitas elaborada neste estudo, utilizando como categorias de estímulos palavras positivas (e.g., honesto, decente e desejável) e negativas (e.g., ruim, reprovável e desprazeroso), e como categorias-alvo monogamia (e.g., monogamia, casamento e exclusividade) e poliamor (e.g., poliamor, paixões e amores). Foram utilizados 40 ensaios para os blocos de treino $(1,2$ e 4$)$ e 60 para os de teste (3 e 5). O FreeIAT, em português, foi instalado em cinco computadores portáteis (laptops) com telas de 14 polegadas, 1280 x 800 pixels de resolução e $60 \mathrm{~Hz}$ de taxa de atualização.

Dados Demográficos e Religiosos. Os participantes responderam a um conjunto de perguntas de natureza demográfica (estado civil, idade, renda e sexo) e religiosa (orientação religiosa e nível de religiosidade).

\section{Procedimento}

Os instrumentos foram administrados por três estudantes do curso de Psicologia devidamente treinados e supervisionados por uma pesquisadora. Estes foram orientados a instruir os participantes acerca de como responder no computador, bem como o formato de resposta dos livretos impressos, cujas medidas foram autoaplicáveis. Os participantes foram informados sobre os propósitos da pesquisa e o caráter voluntário e anônimo de sua colaboração, enfatizando-se que não existiam respostas certas ou erradas. Prévia à sua participação, todos tiveram que ler e assinar um termo de consentimento livre e esclarecido, conforme normas éticas para pesquisas com seres humanos. A aplicação foi feita de forma coletiva, porém os instrumentos foram respondidos individualmente. Os participantes foram reunidos em grupos de dez, metade iniciou respondendo o questionário de autorrelato e os demais principiaram com o TAI. Os bancos de dados dos cinco computadores foram unificados em um único arquivo. Em média, os respondentes gastaram cerca de 10 e 15 minutos para responder o livreto e o TAI, respectivamente.

\section{Resultados}

A análise das medidas explícitas indicaram índices baixos de atitudes favoráveis frente ao poliamor. No caso do fator relacionamento poliamorista, este apresentou um índice de neutralidade, visto que seu valor médio ( $m=3,4$, $D P=0,55)$ ficou acima do ponto mediano da escala de resposta [3 = Não concordo nem discordo; $t(48)=5,19, p$ $<0,001]$. Por outro lado, no fator sentimento poliamorista foi observado um índice predominantemente de discordância desta atitude, cujo valor médio $(1,92, D P=0,76)$ foi inferior ao ponto mediano de sua escala de resposta $[t$ $(48)=9,99, p<0,001]$.

O TAI apresentou como bloco compativel a associação entre as categorias palavras positivas e monogamia, com latência média de associação de 1.208,02 ms ( $D P=$ $364,70)$; o bloco incompativel foi identificado na associação entre palavras positivas e poliamor, com tempo médio de $1.619,59 \mathrm{~ms}(D P=565,76)[t(49)=7,75, p$ $<0,001]$. O escore TAI no formato convencional foi calculado pela diferença entre os blocos incompatível e compativel, resultando em tempo médio de 411,56 ms (DP $=375,63)$. Para conhecer a significância do escore convencional, calculou-se um teste $t$ de Student com o valor zero (ausência de diferença) como critério. O resultado $[t(49)=7,75, p<0,001]$ indicou que este escore diferiu de zero. Nestas análises foram utilizados os valores que apresentaram a média corrigida do tempo de resposta (em ms) dos Blocos 3 e 5, utilizando a substituição de itens perdidos, correspondendo, respectivamente, às colunas três e quatro do output.

Utilizando o Escore $D$, identificou-se uma associação de força moderada $(m=0,43, D P=0,37)$ entre as categorias do bloco congruente (palavras positivas e monogamia). No geral, as análises mostraram uma correlação entre 
Gouveia, V. V., Mendes, L. A. C., Freire, S. E. A., Freires, L. A. \& Barbosa, L. H. G. M. (2014). Medindo Associação Implícita com o FreeIAT em Português: Um Exemplo com Atitudes Implícitas frente ao Poliamor.

as medidas explícita (EAFP) e implícita (TAI Monogamia-Poliamor), pois as atitudes de autorrelato indicaram baixos índices de atitudes frente ao poliamor, da mesma forma, a medida implícita indicou como bloco incongruente (que requer maior tempo) a associação entre Palavras Positivas e Poliamor, sugerindo uma maior dificuldade para a associação entre esses termos. Estes resultados podem ser observados na Tabela 1.

Tabela 1

Média, Desvio Padrão e Correlação entre as Medidas Implícitas e Explícitas

\begin{tabular}{lccccccc}
\hline & $M$ & $D P$ & 1 & 2 & 3 & 4 & 5 \\
\hline 1. Escore-D & 0,43 & 0,37 & & & & & \\
2. Bloco Compatível (ms) & 1208,02 & 364,70 & $-0,19 \dagger$ & & & & \\
3. Bloco Incompatível (ms) & 1619,59 & 565,76 & $0,40^{* *}$ & $0,76^{* * *}$ & & & \\
4. Escore Convencional & 411,56 & 375,63 & $0,78^{* * *}$ & 0,17 & $0,77^{* * *}$ & & \\
5. Relacionamento Poliamorista & 3,40 & 0,55 & $-0,11$ & $-0,11$ & $-0,27^{*}$ & $-0,23 \dagger$ & \\
6. Sentimento Poliamorista & 1,92 & 0,76 & $-0,11$ & $-0,12$ & $-0,30^{*}$ & $-0,34^{* *}$ & $0,47^{* * *}$ \\
\hline
\end{tabular}

$\dagger p<0,10, * p<0,05, * * p<0,01, * * * p<0,001$ (teste unicaudal).

A análise de Pearson apresentou uma correlação positiva $(r=0,78, p<0,01)$ entre os Escores D e o Convencional, indicando uma forte relação entre esses índices de mensuração implícita. Uma das hipóteses para essa forte relação entre os escore é a homogeneidade da amostra, composta de estudantes, com pouca diferenciação de faixa etária, como descrito anteriormente. Segundo Greenwald et al. (2003), os maiores desvios entre esses escores se verificam quando existe diferenciação de habilidades na execução do teste (e.g., TAI executado por jovens em comparação com aquele respondido por pessoas de maior idade).

Considerando que o Escore $D$ positivo indicou uma relação entre Palavras Positivas e a Monogamia, verificou-se uma correlação positiva entre esse índice o tempo do Bloco Incongruente $(r=0,40, p<0,01)$, significando que quanto mais forte a atitude implícita frente à monogamia mais tempo é necessário para associar palavras positivas e o poliamor, isto é, mais lenta é a associação. O mesmo sentido de correlação foi encontrado entre o tempo do Bloco Incongruente com o Escore Convencional $(r=0,77, p<$ $0,01)$ e a latência do Bloco Congruente $(r=0,76, p<0,01)$, estes dois relacionados às atitudes frente à Monogamia.

$\mathrm{Na}$ análise das medidas de autorrelato, neste caso os fatores de Relacionamento e Sentimento Poliamorista, foi identificada uma correlação positiva entre elas $(r=0,47$, $p<0,01)$, o que parece intuitivamente congruente. No caso, pessoas com atitudes favoráveis frente a relacionamentos poliamoristas são mais propensas a sentimentos poliamoristas e vice-versa, sugerindo coerência no sistema atitudinal das pessoas (Maio \& Haddock, 2010).

Quanto à relação entre as medidas implícitas e explícitas, constatou-se uma correlação negativa $(r=-0,34, p$ $<0,05)$ entre os Sentimentos Poliamoristas e o Escore Convencional, apontando que quanto maior as atitudes explícitas frente ao poliamor menor as atitudes implícitas frente à monogamia. Nesse mesmo sentido foi encontrada uma correlação negativa entre os Sentimentos Poliamoristas e a latência do Bloco Incompativel $(r=-0,30, p<0,05)$, demonstrando que quanto maior as atitudes explícitas frente ao poliamor menor o tempo de associação entre as Palavras Positivas e o Poliamor.

No que se refere aos Relacionamentos Poliamoristas, encontrou-se correlação negativa com o tempo do Bloco Incompativel $(r=-0,27, p=0,07)$ e o Escore Convencional $(r=-0,23, p=0,11)$. Apesar de os resultados não serem estatisticamente significativos (Gouveia, Santos, \& Milfont, 2009), cabe observar que seguem a mesma direção daqueles que dizem respeito aos Sentimentos Poliamoristas, em que quanto mais positivas as atitudes frente ao poliamor, menor o tempo necessário para associar $o$ poliamor às palavras positivas.

Ressalta-se que não foram encontradas correlações significativas entre as medidas explícitas com o Escore $D$ e a latência do Bloco Compatível. Esses dois índices estão diretamente ligados à relação entre Palavras Positivas e Monogamia, o que pode indicar que as atitudes frente ao Poliamor não são necessariamente opostas àquelas frente à monogamia, ou seja, uma pessoa que aceita o poliamor pode não ser contrária à monogamia.

\section{Discussão e Direções Futuras}

Conforme previamente indicado, o propósito deste estudo foi introduzir o FreeIAT, mostrando sua utilidade como uma ferramenta para construir medidas implícitas, exemplificando com o construto poliamor. Diante dos resultados, confia-se ter demonstrado sua efetividade. É possível destacar como vantagens deste programa ser uma interface simplificada, o que facilita sua configuração passo a passo em poucas telas, a aplicação do teste em si e o atendimento das variações possíveis do TAI clássico. Além destes atributos, trata-se de um programa gratuito, podendo 
ser aplicado de forma coletiva quando instalado em várias máquinas ao mesmo tempo, sem a necessidade de conexão à internet. Pode-se citar como limitação a não realização de outros modelos de TAI, como o Single Category IAT (Karpinski \& Steinman, 2006), e a impossibilidade de uso on-line ou em rede.

Os instrumentos utilizados no teste indicaram uma relação entre as medidas implícitas e explícitas, principalmente entre as atitudes explícitas frente ao Poliamor e a latência do Bloco Incongruente do teste TAI Monogamia-Poliamor, mostrando que quanto maior as atitudes explícitas frente ao poliamor menor o tempo necessário para associar as palavras positivas e o poliamor, o que era esperado (Greenwald et al., 2003). Porém, não foram encontradas correlações entre o Escore $D$ e as medidas explícitas, o que pode indicar que pessoas favoráveis ao poliamor não são totalmente contrárias à monogamia. Quiçá este resultado pode ser justificado pelo fato de as pessoas considerarem o poliamor como mais uma modalidade de relacionamento que se encontra disponível além da monogamia, por isso o fato de não serem contrárias à monogamia.

Com respeito ao anteriormente indicado, McLuskey (2009), com o objetivo de conhecer como as pessoas constroem suas relações poliamorosas no meio de uma cultura predominantemente monogâmica heteronormativa, observou que elas criam seu mundo a partir do que veem ao seu redor e das opções que se encontram disponíveis, indicando como a invisibilidade das opções as limita a poder escolher uma vida adequada para elas. Neste contexto, há que indicar que não necessariamente os participantes do presente estudo eram contrários à monogamia; talvez eles só não contavam com opções de escolhas para viver suas relações afetivas de forma satisfatória, incluindo o poliamor. De fato, este é um tipo de relacionamento que se constitui como uma alternativa recente (Anapol, 2010; Wolfe, 2005), cujas implicações estão ainda sendo estudadas.

As limitações do presente estudo estão relacionadas ao fato de ser uma aplicação inicial tanto do software quanto das medidas implícitas, necessitando ser expandido para amostras com caracterizações mais variadas. Dessa forma, sugere-se restrição quanto à generalização dos resultados, necessitando de outros estudos que os corroborem. Entretanto, apesar de o tamanho reduzido da amostra $(n=$ 50) ser estimado como um limitador, destaca-se que está dentro do quantitativo comumente admitido em estudos com medidas implícitas (Cvencek, Greenwald, Brown, Gray, \& Snowden, 2010).

Por fim, confia-se que a disponibilização do FreeIAT em português, que mantém sua condição de programa livre de custos, auxilie na propagação das medidas de associação implícita, que já se fazem notar para avaliar alguns construtos (Victoria \& Soares, 2008). Além disso, espera-se que a divulgação desde programa contribua para o seu aperfeiçoamento, inclusive mostrando desdobramentos de seu uso em diversas áreas.

\section{Referências}

Anapol, D. (2010). Polyamory in the twenty-first century: Love and intimacy with multiple partners. Maryland, UK: Rowman \& Littlefield.

Arkes, H. R., \& Tetlock, P. E. (2004). Attributions of implicit prejudice, or "would Jesse Jackson 'fail' the Implicit Association Test?”. Psychological Inquiry, 15, 257-278. doi:10.1207/ s15327965pli1504_01

Ayres, K., Prestwich, A., Conner, M., \& Smith, P. (2011). Emotional eating moderates the relationship between implicit measures of attitudes and chocolate consumption. European Journal of Personality, 25, 317-325. doi:10.1002/per.793

Bargh, J. A. (1994). The four horsemen of automaticity: Awareness, intention, efficiency, and control in social cognition. In R. S. Wyer \& T. K. Srull (Eds.), Handbook of social cognition ( $2^{\text {nd }}$ ed., Vol. 1, pp. 1-40). Hillsdale, NJ: Erlbaum.

Barker, M. (2005). This is my partner and this is my partner's partner: Constructing a polyamourous identity in a monogamous world. Journal of Constructivist Psychology, 18, 75-88. doi:10.1080/10720530590523107

Baron, A. S., \& Banaji, M. R. (2006). The development of implicit attitudes: Evidence of race evaluations from ages 6 and 10 and adulthood. Psychological Science, 17, 53-58. doi:10.1111/j.1467-9280.2005.01664.x

Bassili, J. N., \& Brown, R. (2005). Implicit and explicit attitudes: Research, challenges and theory. In D. Albarracín, B. T. Johnson, \& M. P. Zanna (Eds.), Handbook of attitudes and attitude change (pp. 543-574). Mahwah, NJ: Lawrence Erlbaum.

Blanton, H., \& Jaccard, J. (2006). Arbitrary metrics in psychology. American Psychologist, 61, 27-41. doi:10.1037/0003-066X.61.1.27

Cai, H., Sriram, N., Greenwald, A. G., \& McFarland, S. G. (2004). The implicit association Test's D measure can minimize a cognitive skill confound: Comment on McFarland and Crouch (2002). Social Cognition, 22, 673-684. doi:10.1521/ soco.22.6.673.54821

Clark, A., \& Meade, A. W. (2012). The fakability and validity of an integrity-based IAT. Paper presented at the $27^{\text {th }}$ Annual Meeting of the Society for Industrial and Organizational Psychology, San Diego, CA, USA.

Cvencek, D., Greenwald, A. G., Brown, A. S., Gray, N. S., \& Snowden, R. J. (2010). Faking of the implicit association test is statistically detectable and partly correctable. Basic and Applied Social Psychology, 32, 302-314. doi:10.1080/0197 3533.2010.519236

Cvencek, D., Greenwald, A. G., \& Meltzoff, A. N. (2011). Measuring implicit attitudes of 4-year-old children: The Preschool Implicit Association Test. Journal of Experimental Child Psychology, 109, 187-200. doi:10.1016/j.jecp.2010.11.002

Cvencek, D., Meltzoff, A. N., \& Greenwald, A. G. (2011). Math-gender stereotypes in elementary-school children. Child Development, 82, 766-789. doi:10.1111/j.14678624.2010.01529.x

Fazio, R. H. (1995). Attitudes as object-evaluation associations: Determinants, consequences, and correlates of attitude accessibility. In R. E. Petty \& J. A. Krosnick (Eds.), Attitude strength: Antecedents and consequences (pp. 247-282). Hillsdale, NJ: Lawrence Erlbaum.

Fazio, R. H., \& Olson, M. A. (2003). Implicit measures in social cognition research: Their meaning and use. Annual Review of Psychology, 54, 297-327. doi:10.1146/annurev. psych.54.101601.145225 
Gouveia, V. V., Mendes, L. A. C., Freire, S. E. A., Freires, L. A. \& Barbosa, L. H. G. M. (2014). Medindo Associação Implícita com o FreeIAT em Português: Um Exemplo com Atitudes Implícitas frente ao Poliamor.

Fiedler, K., Messner, C., \& Bluemke, M. (2006). Unresolved problems with the "I", the "A", and the "T": A logical and psychometric critique of the Implicit Association Test (IAT). European Review of Social Psychology, 17, 74-147. doi:10.1080/10463280600681248

Furnham, A. (1986). Response bias, social desirability and dissimulation. Personality and Individual Differences, 7, 385400. doi:10.1016/0191-8869(86)90014-0

Gawronski, B., \& Bodenhausen, G. V. (2006). Associative and propositional processes in evaluation: An integrative review of implicit and explicit attitude change. Psychological Bulletin, 132, 692-731. doi:10.1037/0033-2909.132.5.692

Google Acadêmico. (2012). "Teste de Associação Implícita" e "FreeIAT". Recuperado em 25 de junho, 2012, de http:// scholar.google.com.br

Gouveia, V. V., Athayde, R. A. A., Mendes, L. A. C., \& Freire, S. E. A. (2012). Introdução às medidas implícitas: Conceitos, técnicas e contribuições. Diaphora - Revista da Sociedade de Psicologia do Rio Grande do Sul, 12, 80-92.

Gouveia, V. V., Carvalho, E. A. B., Santos, F. A., \& Almeida, M. R. (2013). Escala Tetrangular do Amor: Testando sua estrutura e invariância fatorial. Psicologia: Ciência e Profissão, 33, 32-45. doi:10.1590/S1414-98932013000100004

Gouveia, V. V., Guerra, V. M., Souza, D. M. F., Santos, W. S., \& Costa, J. M. (2009). Escala de Desejabilidade Social de Marlowe-Crowne: Evidências de sua validade fatorial e consistência interna. Avaliação Psicológica, 8, 87-98.

Gouveia, V. V., Santos, W. S., \& Milfont, T. L. (2009). O uso da estatística na avaliação psicológica: Comentários e considerações práticas. In C. S. Hutz (Ed.), Avanços e polêmicas em avaliação psicológica: Em homenagem a Jurema Alcides Cunha (pp. 127-155). São Paulo, SP: Casa do Psicólogo.

Greenwald, A. G., \& Banaji, M. R. (1995). Implicit social cognition: Attitudes, self-esteem, and stereotypes. Psychology Review, 102, 4-27. doi:10.1037/0033-295X.102.1.4

Greenwald, A. G., Mcghee, D. E., \& Schwartz, J. K. L. (1998). Measuring individual differences in implicit cognition: The implicit association Test. Journal of Personality and Social Psychology, 74, 1464-1480. doi:10.1037/00223514.74.6.1464

Greenwald, A. G., Nosek, B. A., \& Banaji, M. R. (2003). Understanding and using the Implicit Association Test: I. An improved scoring algorithm. Journal of Personality and Social Psychology, 85, 197-216. doi:10.1037/0022-3514.85.2.197

Hartmana, T. K., \& Newmarka, A. J. (2012). Motivated reasoning, political sophistication, and associations between president Obama and Islam. PS: Political Science \& Politics, 45, 449-455. doi:10.1017/S1049096512000327

Hippel, W. V., Brener, L., \& Hippel, C. V. (2008). Implicit prejudice toward injecting drug users predicts intentions to change jobs among drug and alcohol nurses. Psychological Science, 19, 7-11. doi:10.1111/j.1467-9280.2008.02037.x

Jost, J. T., Rudman, L. A., Blair, I. V., Carney, D., Dasgupta, N., Glaser, J., \& Hardin, C. D. (2009). The existence of implicit bias is beyond reasonable doubt: A refutation of ideological and methodological objections and executive summary of ten studies that no manager should ignore. Research in Organizational Behavior, 29, 39-69. doi:10.1016/j.riob.2009.10.001

Karpinski, A., \& Steinman, R. B. (2006). The Single Category Implicit Association Test as a measure of implicit social cognition. Journal of Personality and Social Psychology, 91, 16-32. doi:10.1037/0022-3514.91.1.16
Lane, K. A., Banaji, M. R., Nosek, B. A., \& Greenwald, A. G. (2007). Understanding and using the Implicit Association Test. In B. Wittenbrink \& N. Schwarz (Eds.), Implicit measures of attitudes (pp. 59-102). New York: The Guilford Press.

Leavitt, K., Fong, C. T., \& Greenwald, A. G. (2011). Asking about well-being gets you half an answer: Intra-individual processes of implicit and explicit job attitudes. Journal of Organizational Behavior, 32, 672-687. doi:10.1002/job.746

Lemm, K. M., Lane, K. A., Sattler, D. N., Khan, S., \& Nosek, B. A. (2008). Assessing implicit attitudes with a paper-format Implicit Association Test. In T. G. Morrison \& M. A. Morrison (Eds.), The psychology of modern prejudice (pp. 123-146). Hauppauge, NY: New Science.

Lima, M. E. O., Machado, C., Ávila, J., Lima, C., \& Vala, J. (2006). Normas sociais e preconceito: O impacto da igualdade e da competição no preconceito automático contra os negros. Psicologia: Reflexão e Crítica, 19, 309-319. doi:10.1590/ S0102-79722006000200018

Maio, G. R., \& Haddock, G. (2010). The psychology of attitudes and attitudes change. Los Angeles, CA: Sage.

McLuskey, K. (2009). Polyamory: Constructing relationships outside of monogamy (Master's thesis, Department of Sociology, University of Victoria, Canada).

Meade, A. W. (2009). FreeIAT: An Open-Source Program to Administer the Implicit Association Test. Applied Psychological Measurement, 33, 643-643. doi:10.1177/0146621608327803

Mori, K., Uchida, A., \& Imada, R. (2008). A paper-format group performance test for measuring the implicit association of target concepts. Behavior Research Methods, 40, 546-555. doi:10.3758/BRM.40.2.546

Nosek, B. A., \& Banaji, M. R. (2001). The go no-go association task. Social Cognition, 19, 625-664. doi:10.1521/ soco.19.6.625.20886

Nosek, B. A., Greenwald, A. G., \& Banaji, M. R. (2005). Understanding and using the Implicit Association Test: II. Method variables and construct validity. Personality and Social Psychology Bulletin, 31, 166-180. doi:10.1177/0146167204271418

Nosek, B. A., Greenwald, A. G., \& Banaji, M. R. (2007). The Implicit Association Test at age 7: A methodological and conceptual review. In J. A. Bargh (Ed.), Automatic processes in social thinking and behavior (pp. 265-292). London: Psychology Press.

Nosek, B. A., Hawkins, C. B., \& Frazier, R. S. (2011). Implicit social cognition: From measures to mechanisms. Trends in Cognitive Sciences, 15, 152-159. doi:10.1016/j.tics.2011.01.005

Periódicos Capes. (2012). "Teste de Associação Implícita" e "FreeIAT". Recuperado em 25 de junho, 2012, de www. periodicos.capes.gov.br

Pimentel, C. E., Torres, C. V., \& Günther, H. (2011). Estratégias de mensuração de atitudes em Psicologia Social. In C. V. Torres \& E. R. Neiva (Eds.), Psicologia Social: Principais temas e vertentes (pp. 196-210). Porto Alegre, RS: ArtMed.

Pruett, S. R., \& Chan, F. (2006). The development and psychometric validation of the Disability Attitude Implicit Association Test. Rehabilitation Psychology, 51, 202-213. doi:10.1037/0090-5550.51.3.202

PsycNET. (2012). "Priming”. Recuperado em 26 de junho, 2012, de http://psycnet.apa.org/

Schnabel, K., Asendorpf, J. B., \& Greenwald, A. G. (2008). Implicit Association Tests: A landmark for the assessment of implicit personality self-concept. In G. J. Boyle, G. Matthews, \& D. H. Saklofske (Eds.), Handbook of personality theory and testing (pp. 508-528). London: Sage. 
Seisdedos, N. (1996). The "IM" (impression management) Scale. European Review of Applied Psychology, 46, 45-54.

Sriram, N., \& Greenwald, A. G. (2009). The brief Implicit Association Test. Experimental Psychology, 56, 283-294. doi:10.1027/1618-3169.56.4.283

Steffens, M. C. (2004). Is the Implicit Association Test immune to faking? Experimental Psychology, 51, 165-179. doi:10.1027/1618-3169.51.3.165

Stüttgen, P., Vosgerau, J., Messner, C., \& Boatwright, P. (2011). Adding significance to the Implicit Association Test. Retrieved December, 12, 2011, from http://www.andrew.cmu.edu/user/ pstuettg/Academic_files/IAT.pdf

Victoria, M. S., \& Soares, A. B. (2008). Avaliação do Teste de Associação Implícita numa amostra de estudantes de Psicologia. PSIC - Revista de Psicologia da Vetor Editora, 9, 211-218.

Walker, S. S., \& Schimmack, U. (2008). Validity of a happiness Implicit Association Test as a measure of subjective well-being. Journal of Research in Personality, 42, 490-497. doi:10.1016/j.jrp.2007.07.005

Wittenbrink, B., \& Schwarz, N. (2007). Implicit measures of attitudes. New York: Guilford Press.

Wolfe, L. P. (2005). Jealousy and transformation in polyamorous relationships (Unpublished doctoral dissertation). The Institute for Advanced Study of Human Sexuality, San Francisco, CA, USA.

Wright, N. A., \& Meade, A. W. (2011). Predictive validity and procedural justice of the implicit association test. Paper presented at the $26^{\text {th }}$ Annual Meeting of the Society for Industrial and Organizational Psychology, Chicago, IL, USA.

Wright, N. A., \& Meade, A. W. (2012). An exploration of cognitive ability contamination in the Implicit Association Test methodology. Computers in Human Behavior, 28, 393-399. doi:10.1016/j.chb.2011.10.009 